\title{
NEW WEIGHTED ESTIMATES \\ FOR BILINEAR FRACTIONAL INTEGRAL OPERATORS
}

\author{
KABE MOEN
}

ABSTRACT. We prove a plethora of weighted estimates for bilinear fractional integral operators of the form

$$
B I_{\alpha}(f, g)(x)=\int_{\mathbb{R}^{n}} \frac{f(x-t) g(x+t)}{|t|^{n-\alpha}} d t, \quad 0<\alpha<n .
$$

When the target space has an exponent greater than one, many weighted estimates follow trivially from Hölder's inequality and the known linear theory. We address the case where the target Lebesgue space is at most one and prove several interesting one and two weight estimates. As an application we formulate a bilinear version of the Stein-Weiss inequality for fractional integrals.

\section{INTRODUCTION}

Consider the family of bilinear fractional integral operators

$$
B I_{\alpha}(f, g)(x)=\int_{\mathbb{R}^{n}} \frac{f(x-t) g(x+t)}{|t|^{n-\alpha}} d t, \quad 0<\alpha<n .
$$

Such operators have a long history and were studied by Grafakos [8], Kenig and Stein [11, and Grafakos and Kalton [9], among others. The operators have attracted interest because of their similarity to the bilinear Hilbert transform

$$
B H(f, g)(x)=p \cdot v \cdot \int_{\mathbb{R}} \frac{f(x-t) g(x+t)}{t} d t .
$$

Indeed, when $n=1$, let $k$ and $k_{\alpha}$ be the distributional kernels in $\mathbb{R}^{2}$ of $B H$ and $B I_{\alpha}$ respectively, i.e.,

$$
B H(f, g)(x)=\int_{\mathbb{R}^{2}} k(x-y, x-z) f(y) g(z) d y d z
$$

and

$$
B I_{\alpha}(f, g)(x)=\int_{\mathbb{R}^{2}} k_{\alpha}(x-y, x-z) f(y) g(z) d y d z .
$$

Then we may write

$$
k(u, v)=\frac{\delta(u+v)}{u} \quad \text { and } \quad k_{\alpha}(u, v)=\frac{\delta(u+v)}{|u|^{1-\alpha}},
$$

Received by the editors November 7, 2011.

2010 Mathematics Subject Classification. Primary 42B20, 26A33.

Key words and phrases. Bilinear operators, fractional integration, weighted inequalities.

The author was partially supported by NSF Grant 1201504.

(C) 2013 American Mathematical Society Reverts to public domain 28 years from publication 
where $\delta$ is the point mass measure at the origin. If $B I_{\alpha}$ satisfies any $L^{p_{1}}\left(\mathbb{R}^{n}\right) \times$ $L^{p_{2}}\left(\mathbb{R}^{n}\right) \rightarrow L^{q}\left(\mathbb{R}^{n}\right)$ estimates, then a scaling argument shows that $p_{1}, p_{2}$, and $q$ must satisfy the Sobolev relationship

$$
\frac{1}{q}=\frac{1}{p_{1}}+\frac{1}{p_{2}}-\frac{\alpha}{n}
$$

It was shown in [8, 9, 11] that if $1<p_{1}, p_{2}<\infty$ and $q$ is defined by equation (10), then

$$
B I_{\alpha}: L^{p_{1}}\left(\mathbb{R}^{n}\right) \times L^{p_{2}}\left(\mathbb{R}^{n}\right) \rightarrow L^{q}\left(\mathbb{R}^{n}\right) .
$$

Unlike the bilinear Hilbert transform, $B I_{\alpha}$ is a positive operator in the sense that $\left|B I_{\alpha}(f, g)\right| \leq B I_{\alpha}(|f|,|g|)$. This allows one to work with non-negative functions $f$ and $g$. Moreover, one may trivially obtain $L^{p_{1}}\left(\mathbb{R}^{n}\right) \times L^{p_{2}}\left(\mathbb{R}^{n}\right) \rightarrow L^{q}\left(\mathbb{R}^{n}\right)$ bounds when $q>1$. This follows from the known linear bounds. Indeed, for any pair of conjugate exponents $\frac{1}{r}+\frac{1}{s}=1$, Hölder's inequality yields

$$
B I_{\alpha}(f, g) \leq I_{\alpha}\left(f^{r}\right)^{\frac{1}{r}} I_{\alpha}\left(g^{s}\right)^{\frac{1}{s}},
$$

where

$$
I_{\alpha} f(x)=\left(|\cdot|^{\alpha-n} * f\right)(x)=\int_{\mathbb{R}^{n}} \frac{f(x-y)}{|y|^{n-\alpha}} d y
$$

is the linear fractional integral operator or Riesz potential. If we let $r=p_{1} / p$ and $s=p_{2} / p$, then $r, s>1$ and $1 / r+1 / s=1$. Using the fact that $I_{\alpha}: L^{p}\left(\mathbb{R}^{n}\right) \rightarrow$ $L^{q}\left(\mathbb{R}^{n}\right)$, for $f, g \geq 0$, we have

$$
\begin{aligned}
\left(\int_{\mathbb{R}^{n}}\left(B I_{\alpha}(f, g)\right)^{q} d x\right)^{\frac{1}{q}} & \leq\left(\int_{\mathbb{R}^{n}} I_{\alpha}\left(f^{r}\right)^{\frac{q}{r}} I_{\alpha}\left(g^{s}\right)^{\frac{q}{s}} d x\right)^{\frac{1}{q}} \\
& \leq\left(\int_{\mathbb{R}^{n}} I_{\alpha}\left(f^{r}\right)^{q} d x\right)^{\frac{1}{q r}}\left(\int_{\mathbb{R}^{n}} I_{\alpha}\left(g^{s}\right)^{q} d x\right)^{\frac{1}{s q}} \\
& \leq C\left(\int_{\mathbb{R}^{n}} f^{p_{1}} d x\right)^{\frac{1}{p_{1}}}\left(\int_{\mathbb{R}^{n}} g^{p_{2}} d x\right)^{\frac{1}{p_{2}}} .
\end{aligned}
$$

Moreover, this exact line of reasoning shows that weighted estimates of the form

$$
\begin{aligned}
& \left(\int_{\mathbb{R}^{n}}\left(B I_{\alpha}(f, g) w_{1} w_{2}\right)^{q} d x\right)^{\frac{1}{q}} \\
& \quad \leq C\left(\int_{\mathbb{R}^{n}}\left(f w_{1}\right)^{p_{1}} d x\right)^{\frac{1}{p_{1}}}\left(\int_{\mathbb{R}^{n}}\left(g w_{2}\right)^{p_{2}} d x\right)^{\frac{1}{p_{2}}}
\end{aligned}
$$

hold when $q>1$ (see also [10] for similar bounds that follow from Hölder's inequality). Muckenhoupt and Wheeden [19] showed that for $1 / q=1 / p-\alpha / n$

$$
\left(\int_{\mathbb{R}^{n}}\left(\left(I_{\alpha} f\right) w\right)^{q} d x\right)^{\frac{1}{q}} \leq C\left(\int_{\mathbb{R}^{n}}(f w)^{p} d x\right)^{\frac{1}{p}}
$$

if and only if $w \in A_{p, q}$

$$
[w]_{A_{p, q}}=\sup _{Q}\left(\frac{1}{|Q|} \int_{Q} w^{q} d x\right)^{\frac{1}{q}}\left(\frac{1}{|Q|} \int_{Q} w^{-p^{\prime}} d x\right)^{\frac{1}{p^{\prime}}}<\infty .
$$


Using inequality (2), it was observed in [2] that for $1<p_{1}, p_{2}, p, q<\infty$ satisfying (11), then (4) holds when $w_{1}, w_{2} \in A_{p, q}$. Using inequality (2) and the linear theory once again, one may derive a variety of two weight (really three weight) inequalities of the form

$$
\begin{aligned}
& \left(\int_{\mathbb{R}^{n}}\left(B I_{\alpha}(f, g) u\right)^{q} d x\right)^{1 / q} \\
& \quad \leq C\left(\int_{\mathbb{R}^{n}}\left(f v_{1}\right)^{p_{1}} d x\right)^{1 / p_{1}}\left(\int_{\mathbb{R}^{n}}\left(g v_{2}\right)^{p_{2}} d x\right)^{1 / p_{2}} ;
\end{aligned}
$$

for example, if the pairs of weights $\left(u, v_{1}\right)$ and $\left(u, v_{2}\right)$ individually satisfy Sawyer's testing conditions [23] or certain bump conditions [20]. We emphasize that in order to use the Hölder type bounds in (2) one must have $q>1$ and the pairs $\left(u, v_{1}\right)$, $\left(u, v_{2}\right)$ must independently satisfy some sort of two weight condition.

All of the above estimates follow from two things: the target Lebesgue space having exponent larger than one and the known linear theory. An important feature of bilinear operators is that they map into Lebesgue spaces with exponent less than one. To the best of the author's knowledge there are no known weighted estimates for the operators $B H, B I_{\alpha}$ or their related maximal operators when the target space has exponent less than one. One possible reason for this is that the above strategy completely fails. The bilinear operator $(f, g) \mapsto I_{\alpha}\left(f^{s}\right)^{1 / s} I_{\alpha}\left(g^{r}\right)^{1 / r}$ cannot map into $L^{q}\left(\mathbb{R}^{n}\right)$ with $q \leq 1$, because this would imply that the linear operator $I_{\alpha}$ does the same by taking $f^{s}=g^{r}$.

The case when the target space has exponent less than one has drawn interest because of its complications. For example, the bilinear Hilbert transform and related bilinear maximal function satisfy $L^{p_{1}}(\mathbb{R}) \times L^{p_{2}}(\mathbb{R}) \rightarrow L^{p}(\mathbb{R})$ bounds for some $1 / p=1 / p_{1}+1 / p_{2}$. If $1<p_{1}, p_{2}<\infty$, then $p$ should range all the way down to $1 / 2$, but the known techniques only imply $p>2 / 3$ (see [12 14]).

Below, we study both one weight (4) and two weight (5) estimates for $B I_{\alpha}$ when $q \leq 1$. Our results are new and provide the first non-trivial weighted estimates for $B I_{\alpha}$ and the only known weighted estimates for $B I_{\alpha}$ when $q \leq 1$. The estimates we obtain parallel earlier results by the author [17] and by Chen and Xue [3] for the less singular bilinear fractional integral operator

$$
\mathcal{I}_{\alpha}(f, g)(x)=\int_{\mathbb{R}^{2 n}} \frac{f(y) g(z)}{(|x-y|+|x-z|)^{2 n-\alpha}} d y d z .
$$

Notice that the kernel of $\mathcal{I}_{\alpha}$,

$$
K_{\alpha}(u, v)=(|u|+|v|)^{-n+\alpha},
$$

has a singularity at the origin in $\mathbb{R}^{2 n}$ as opposed to the kernel of $B I_{\alpha}$,

$$
k_{\alpha}(u, v)=\frac{\delta(u+v)}{|u|^{n-\alpha}},
$$

which has a singularity along a line. For $\mathcal{I}_{\alpha}$ a complete weighted theory was developed in [17. The theory for $\mathcal{I}_{\alpha}$ has expanded in several directions, including spaces of homogeneous type [16, pointwise and norm estimates with various maximal functions [22, and multilinear commutators [1], to name a few. Moreover, it has given rise to several applications including Poincaré and Sobolev inequalities for products of functions and Leibniz type rules in various function spaces (see $[2,16,18])$. 
To put our results for $B I_{\alpha}$ in context we briefly recall the results for $\mathcal{I}_{\alpha}$. The classes of weights $\left(u, v_{1}, v_{2}\right)$ that imply bounds for $\mathcal{I}_{\alpha}$ are different, depending on whether $q>1$ or $q \leq 1$. We focus on $q \leq 1$.

Theorem A ([17]). Suppose $1<p_{1}, p_{2}<\infty$ and $1 / 2<p=\frac{p_{1} p_{2}}{p_{1}+p_{2}} \leq q \leq 1$. If $\left(u, v_{1}, v_{2}\right)$ is a pair of weights that, for some $r>1$, satisfies

$$
\sup _{Q}|Q|^{\frac{\alpha}{n}+\frac{1}{q}-\frac{1}{p}}\left(\frac{1}{|Q|} \int_{Q} u^{q} d x\right)^{\frac{1}{q}} \prod_{i=1}^{2}\left(\frac{1}{|Q|} \int_{Q} v_{i}^{-r p_{i}^{\prime}} d x\right)^{\frac{1}{r p_{i}^{\prime}}}<\infty,
$$

then for $f, g \geq 0$,

$$
\begin{aligned}
& \left(\int_{\mathbb{R}^{n}}\left(\mathcal{I}_{\alpha}(f, g) u\right)^{q} d x\right)^{\frac{1}{q}} \\
& \quad \leq C\left(\int_{\mathbb{R}^{n}}\left(f v_{1}\right)^{p_{1}} d x\right)^{\frac{1}{p_{1}}}\left(\int_{\mathbb{R}^{n}}\left(g v_{2}\right)^{p_{2}} d x\right)^{\frac{1}{p_{2}}} .
\end{aligned}
$$

Actually a weaker hypothesis than (6) in the setting of Orlicz spaces implies the conclusion (see Section 2). There is also a version of Theorem $\mathrm{A}$ when $q>1$ that requires a bump condition on the weight $u$. For the operator $B I_{\alpha}$ we will need to bump the weight $u$ as well (see Theorem 1.1 and Remark 1.3 below).

For one weight inequalities, that is, pairs of weights $\left(w_{1}, w_{2}\right)$ that satisfy

$$
\begin{aligned}
& \left(\int_{\mathbb{R}^{n}}\left(\mathcal{I}_{\alpha}(f, g) w_{1} w_{2}\right)^{q} d x\right)^{\frac{1}{q}} \\
& \quad \leq C\left(\int_{\mathbb{R}^{n}}\left(f w_{1}\right)^{p_{1}} d x\right)^{\frac{1}{p_{1}}}\left(\int_{\mathbb{R}^{n}}\left(g w_{2}\right)^{p_{2}} d x\right)^{\frac{1}{p_{2}}},
\end{aligned}
$$

there is a complete characterization. In this case, again, a simple scaling argument shows that we must have $1<p_{1}, p_{2}<\infty$ and $q$ satisfy (1).

Theorem B ([17]). Suppose $1<p_{1}, p_{2}<\infty$ and $q>p=\frac{p_{1} p_{2}}{p_{1}+p_{2}}$ is defined by (11). Then $\mathcal{I}_{\alpha}$ satisfies (7) if and only if $\left(w_{1}, w_{2}\right) \in A_{\left(p_{1}, p_{2}\right), q}$ :

$$
\sup _{Q}\left(\frac{1}{|Q|} \int_{Q}\left(w_{1} w_{2}\right)^{q}\right)^{\frac{1}{q}}\left(\frac{1}{|Q|} \int_{Q} w_{1}^{-p_{1}^{\prime}}\right)^{\frac{1}{p_{1}^{\prime}}}\left(\frac{1}{|Q|} \int_{Q} w_{2}^{-p_{2}^{\prime}}\right)^{\frac{1}{p_{2}^{\prime}}}<\infty .
$$

The class $A_{\left(p_{1}, p_{2}\right), q}$ is a rather large class of weights with several interesting properties (see Section 3 of [17]). In particular the weights $\left(w_{1} w_{2}\right)^{q}, w_{1}^{-p_{1}^{\prime}}$, and $w_{2}^{-p_{2}^{\prime}}$ are $A_{\infty}$ weights.

We now state our main results for $B I_{\alpha}$. For two weight inequalities we have the following.

Theorem 1.1. Suppose $1<p_{1}, p_{2}<\infty$ and $1 / 2<\frac{p_{1} p_{2}}{p_{1}+p_{2}} \leq q \leq 1$. If $\left(u, v_{1}, v_{2}\right)$ are weights that satisfy

$$
\sup _{Q}|Q|^{\frac{\alpha}{n}+\frac{1}{q}-\frac{1}{p}}\left(\frac{1}{|Q|} \int_{Q} u^{\frac{q}{1-q}}\right)^{\frac{1-q}{q}} \prod_{i=1}^{2}\left(\frac{1}{|Q|} \int_{Q} v_{i}^{-r p_{i}^{\prime}}\right)^{\frac{1}{r p_{i}^{\prime}}}<\infty,
$$



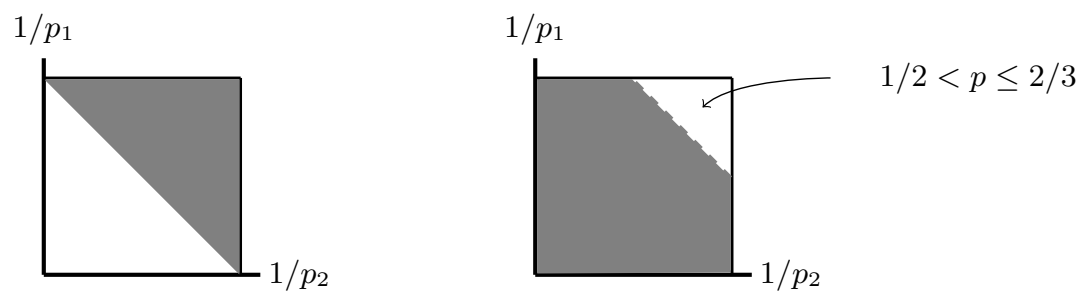

FiguRE 1 . The shaded areas represent the range of $p_{1}, p_{2}$ to obtain weighted estimates for $B I_{\alpha}$ and the known $L^{p_{1}} \times L^{p_{2}} \rightarrow L^{p}$ bounds for $B H$ respectively.

where $\left(f_{Q} u^{\frac{q}{1-q}} d x\right)^{\frac{1-q}{q}}=\sup _{Q} u$ when $q=1$, then for $f, g \geq 0$,

$$
\left(\int_{\mathbb{R}^{n}}\left(B I_{\alpha}(f, g) u\right)^{q} d x\right)^{\frac{1}{q}} \leq C\left(\int_{\mathbb{R}^{n}}\left(f v_{1}\right)^{p_{1}} d x\right)^{\frac{1}{p_{1}}}\left(\int_{\mathbb{R}^{n}}\left(g v_{2}\right)^{p_{2}} d x\right)^{\frac{1}{p_{2}}} .
$$

Remark 1.2. First notice that the range on $p_{1}, p_{2}$ is restricted, since ultimately $1 / p_{1}+1 / p_{2} \geq 1$. However, this is the interesting range, where $p_{1}, p_{2}$ can be close to one (see Figure 1).

Remark 1.3. In Theorem $\mathrm{A}$ we did not require a bump on the weight $u$. That is, the average of $u$ appearing in condition (6) is simply an $L^{q}$ average, whereas the average of $u$ appearing in condition (8) of Theorem 1.1 is an $L^{r q}$ average with $r=(1-q)^{-1}$. Roughly speaking, this extra integrability on $u$ is used to absorb the more singular nature of $B I_{\alpha}$.

We also prove the conclusion of Theorem 1.1 under much weaker assumptions on the weights $v_{1}$ and $v_{2}$. This condition is in the setting of Orlicz spaces whose Young functions satisfy the $B_{p}$ condition [20]. We state the theorem below in the context of $L(\log L)$ type bumps, and refer the reader to Section 2 for pertinent definitions and to Section 3 for the most general result, Theorem 3.3 .

Theorem 1.4. Suppose $1<p_{1}, p_{2}<\infty$ and $q \leq 1$ are such that $\frac{p_{1} p_{2}}{p_{1}+p_{2}} \leq q \leq 1$. If $\left(u, v_{1}, v_{2}\right)$ are weights satisfying

$$
\sup _{Q}|Q|^{\frac{\alpha}{n}+\frac{1}{q}-\frac{1}{p}}\left(\frac{1}{|Q|} \int_{Q} u^{\frac{q}{1-q}}\right)^{\frac{1-q}{q}} \prod_{i=1}^{2}\left\|v_{i}^{-1}\right\|_{L^{p_{i}^{\prime}(\log L)^{p_{i}^{\prime}-1+\delta}\left(Q, \frac{d x}{|Q|}\right)}}<\infty
$$

where $\left(\frac{1}{|Q|} \int_{Q} u^{\frac{q}{1-q}} d x\right)^{\frac{1-q}{q}}=\sup _{Q} u$ when $q=1$, then

$$
\left(\int_{\mathbb{R}^{n}}\left(B I_{\alpha}(f, g) u\right)^{q} d x\right)^{\frac{1}{q}} \leq C\left(\int_{\mathbb{R}^{n}}\left(f v_{1}\right)^{p_{1}} d x\right)^{\frac{1}{p_{1}}}\left(\int_{\mathbb{R}^{n}}\left(g v_{2}\right)^{p_{2}} d x\right)^{\frac{1}{p_{2}}}
$$

for $f, g \geq 0$.

For one weight inequalities we take $1 / q=1 / p-\alpha / n$ and $u=w_{1} w_{2}$ to arrive at the following theorem. 


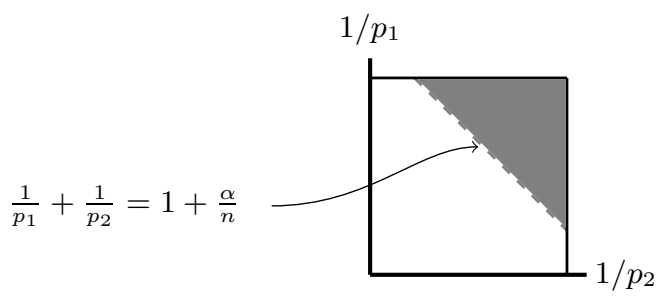

FiguRE 2. The shaded areas represent the range of $p_{1}, p_{2}$ to obtain one weight estimates for $B I_{\alpha}$.

Theorem 1.5. Suppose $1<p_{1}, p_{2}<\infty, \frac{1}{p_{1}}+\frac{1}{p_{2}}>1+\frac{\alpha}{n}$ and $q$ satisfies (11). If $\left(w_{1}, w_{2}\right) \in A_{\left(p_{1}, p_{2}\right), \tilde{q}}$ where $\tilde{q}=q /(1-q)$, i.e.,

$$
\sup _{Q}\left(\frac{1}{|Q|} \int_{Q}\left(w_{1} w_{2}\right)^{\tilde{q}}\right)^{\frac{1}{\tilde{q}}}\left(\frac{1}{|Q|} \int_{Q} w_{1}^{-p_{1}^{\prime}}\right)^{\frac{1}{p_{1}^{\prime}}}\left(\frac{1}{|Q|} \int_{Q} w_{2}^{-p_{2}^{\prime}}\right)^{\frac{1}{p_{2}^{\prime}}}<\infty,
$$

then for $f, g \geq 0$,

$$
\begin{aligned}
& \left(\int_{\mathbb{R}^{n}}\left(B I_{\alpha}(f, g) w_{1} w_{2}\right)^{q} d x\right)^{\frac{1}{q}} \\
& \quad \leq C\left(\int_{\mathbb{R}^{n}}\left(f w_{1}\right)^{p_{1}} d x\right)^{\frac{1}{p_{1}}}\left(\int_{\mathbb{R}^{n}}\left(g w_{2}\right)^{p_{2}} d x\right)^{\frac{1}{p_{2}}} .
\end{aligned}
$$

Remark 1.6. The class $A_{\left(p_{1}, p_{2}\right), q}$ is a rather large class of weights. In fact, it follows from Hölder's inequality that

$$
\bigcup_{q_{1}, q_{2}: \frac{1}{q_{1}}+\frac{1}{q_{2}}=\frac{1}{q}} A_{q_{1}, p_{1}} \times A_{p_{2}, q_{2}} \subsetneq A_{\left(p_{1}, p_{2}\right), q}
$$

(see [17 for details). However, we do not know if (9) is a necessary condition.

Remark 1.7. The range $\frac{1}{p_{1}}+\frac{1}{p_{2}}>1+\frac{\alpha}{n}$ is once again an interesting range because $p_{1}$ and $p_{2}$ can be close to one (see Figure 2). By taking $w_{1}=w_{2}=1$ in Theorem 1.5. we obtain a new proof (one that does not use bilinear interpolation) of the results of Grafakos and Kalton [9] and Kenig and Stein [11] when $q \leq 1$.

We will also be concerned with weighted control of $B I_{\alpha}$ by various fractional maximal operators. To introduce this notion we recall the linear results. The fractional maximal operator,

$$
M_{\alpha} f(x)=\sup _{Q \ni x} \frac{|Q|^{\frac{\alpha}{n}}}{|Q|} \int_{Q}|f(t)| d t,
$$

was introduced by Muckenhoupt and Wheeden to study $I_{\alpha}$. When $\alpha=0, M_{0}=M$ is the Hardy-Littlewood maximal operator. A simple computation, when $0<\alpha<$ $n$, shows that

$$
M_{\alpha} f(x) \leq c I_{\alpha} f(x) .
$$

In particular, $M_{\alpha}$ is bounded whenever $I_{\alpha}$ is bounded. However, much more is true. Muckenhoupt and Wheeden [19] showed that for all $p, 0<p<\infty$ and all $w \in A_{\infty}$,

$$
\int_{\mathbb{R}^{n}}\left|I_{\alpha} f\right|^{p} w d x \leq C \int_{\mathbb{R}^{n}}\left(M_{\alpha} f\right)^{p} w d x .
$$


Below we prove similar results for $B I_{\alpha}$.

We begin with two related fractional bilinear maximal operators. The first is the natural fractional maximal function for $B I_{\alpha}$, given by

$$
B M_{\alpha}(f, g)(x)=\sup _{r>0} \frac{1}{(2 r)^{n-\alpha}} \int_{|t|_{\infty} \leq r}|f(x-t) g(x+t)| d t .
$$

A simple computation proved in [7] shows that for $0<\alpha<n$,

$$
B M_{\alpha}(f, g)(x) \leq c B I_{\alpha}(f, g)(x) .
$$

Thus any estimates we prove for $B I_{\alpha}$ hold for $B M_{\alpha}$ as well. In particular, Theorems 1.1, 1.4 and 1.5 all hold for $B M_{\alpha}$ when $\alpha>0$. When $\alpha=0$, as mentioned in the introduction, much less is known [12.

A family of fractional maximal operators of a less singular nature was introduced in [17:

$$
\mathcal{M}_{\alpha}(f, g)(x)=\sup _{Q \ni x}|Q|^{\frac{\alpha}{n}} \frac{1}{|Q|} \int_{Q} f d x \cdot \frac{1}{|Q|} \int_{Q} g d x, \quad 0<\alpha<2 n .
$$

When $\alpha=0, \mathcal{M}_{0}$ is a bilinear maximal operator introduced in [15]. $\mathcal{M}$ is used to study weighted inequalities for multilinear Calderón-Zygmund operators. $\mathcal{M}_{\alpha}$ is to $\mathcal{I}_{\alpha}$ as $B M_{\alpha}$ is to $B I_{\alpha}$. However, $\mathcal{M}_{\alpha}$ is much simpler to work with. One can obtain the entire range of $\mathcal{M}_{\alpha}: L^{p_{1}}\left(\mathbb{R}^{n}\right) \times L^{p_{2}}\left(\mathbb{R}^{n}\right) \rightarrow L^{q}\left(\mathbb{R}^{n}\right)$ bounds simply from the pointwise inequality

$$
\mathcal{M}_{\alpha}(f, g)(x) \leq M_{\alpha_{1}}(f)(x) M_{\alpha_{2}}(g)(x), \quad \alpha_{1}+\alpha_{2}=\alpha .
$$

Moreover, it was shown in [17] that given any $0<p<\infty$ and $w \in A_{\infty}$ there exist constants $c$ and $C$ such that

$$
c\left\|\mathcal{M}_{\alpha}(f, g)\right\|_{L^{p}(w)} \leq\left\|\mathcal{I}_{\alpha}(f, g)\right\|_{L^{p}(w)} \leq C\left\|\mathcal{M}_{\alpha}(f, g)\right\|_{L^{p}(w)} .
$$

Below we investigate the relationship between $B M_{\alpha}, B I_{\alpha}$, and $\mathcal{M}_{\alpha}$. We begin with a couple of simple observations. Fix $x \in \mathbb{R}$ and let $Q$ be a cube containing $x$ with side length $\ell(Q)$ and notice that

$$
\frac{1}{|Q|} \int_{Q} \frac{1}{\ell(Q)^{n-\alpha}} \int_{|t|_{\infty} \leq \frac{\ell(Q)}{2}} f(y-t) g(y+t) d t d y \leq \frac{1}{|Q|} \int_{Q} B M_{\alpha}(f, g)(y) d y .
$$

On the other hand using the change of variables $r=y-t, s=y+t$ (see Figure 3) we have

$$
\begin{array}{r}
\frac{1}{|Q|} \int_{Q} \frac{1}{\ell(Q)^{n-\alpha}} \int_{|t|_{\infty} \leq \frac{\ell(Q)}{2}} f(y-t) g(y+t) d t d y \\
\geq c|Q|^{\frac{\alpha}{n}} \frac{1}{|Q|} \int_{Q} f d r \cdot \frac{1}{|Q|} \int_{Q} g d s .
\end{array}
$$

Taking the supremum over all such cubes we have

$$
\mathcal{M}_{\alpha}(f, g)(x) \leq c M\left(B M_{\alpha}(f, g)\right)(x) ;
$$

note that this inequality holds for $\alpha=0$ as well. From here one can see that

$$
\left\|\mathcal{M}_{\alpha}(f, g)\right\|_{L^{q}\left(\mathbb{R}^{n}\right)} \leq c\left\|B M_{\alpha}(f, g)\right\|_{L^{q}\left(\mathbb{R}^{n}\right)} \leq c\left\|B I_{\alpha}(f, g)\right\|_{L^{q}\left(\mathbb{R}^{n}\right)},
$$

for $1<q<\infty$. However, it is more interesting to see when the opposite inequality holds; that is, can we dominate $B M_{\alpha}$ or $B I_{\alpha}$ in norm by the simpler operator $\mathcal{M}_{\alpha}$ ? Below we provide an affirmative answer to this question when $0<q \leq 1$. 
Before we state our results for $B I_{\alpha}$ we need to introduce the reverse Hölder class of weights. Given $r, 1<r \leq \infty$, we say that $w \in R H_{r}$ if there exists a constant $C$ such that

$$
\left(\frac{1}{|Q|} \int_{Q} w^{r} d x\right)^{\frac{1}{r}} \leq \frac{C}{|Q|} \int_{Q} w d x
$$

for all cubes $Q$. We are now ready to state our weighted control theorem for $B I_{\alpha}$.

Theorem 1.8. Suppose $0<\alpha<n, 0<q \leq 1$, and $w \in R H_{(1 / q)^{\prime}}$. Then

$$
\int_{\mathbb{R}^{n}}\left|B I_{\alpha}(f, g)\right|^{q} w d x \leq C \int_{\mathbb{R}^{n}}\left|\mathcal{M}_{\alpha}(f, g)\right|^{q} w d x .
$$

In particular, when $w=1$ and $0<q \leq 1$ there exist constants $c$ and $C$ such that

$$
c\left\|B M_{\alpha}(f, g)\right\|_{L^{q}\left(\mathbb{R}^{n}\right)} \leq\left\|B I_{\alpha}(f, g)\right\|_{L^{q}\left(\mathbb{R}^{n}\right)} \leq C\left\|\mathcal{M}_{\alpha}(f, g)\right\|_{L^{q}\left(\mathbb{R}^{n}\right)} .
$$

Remark 1.9. We do not know if $\left\|B I_{\alpha}(f, g)\right\|_{L^{q}\left(\mathbb{R}^{n}\right)}$ is controlled by $\left\|B M_{\alpha}(f, g)\right\|_{L^{q}\left(\mathbb{R}^{n}\right)}$ or whether $B M_{\alpha}$ or $B I_{\alpha}$ dominate $\mathcal{M}_{\alpha}$ pointwise or in norm. Moreover, we do not know if we may take $\alpha=0$ in the inequality

$$
\left\|B M_{\alpha}(f, g)\right\|_{L^{q}\left(\mathbb{R}^{n}\right)} \leq C\left\|\mathcal{M}_{\alpha}(f, g)\right\|_{L^{q}\left(\mathbb{R}^{n}\right)} .
$$

For our techniques, the constant $C$ depends on $\alpha$ and blows up as $\alpha \rightarrow 0$ because of the intermediate estimate involving $B I_{\alpha}$. If one was able to take $\alpha=0$ (which would necessarily require one to remove the intermediate estimate involving $B I_{\alpha}$ ), then, dropping the $\alpha=0$ subscript, it would follow that $B M: L^{p_{1}}\left(\mathbb{R}^{n}\right) \times L^{p_{2}}\left(\mathbb{R}^{n}\right) \rightarrow$ $L^{p}\left(\mathbb{R}^{n}\right)$ for all $1<p_{1}, p_{2}<\infty$ and $p$ satisfying $\frac{1}{p_{1}}+\frac{1}{p_{2}}=\frac{1}{p}$. In particular $p>1 / 2$.

The highlights of the paper are as follows. In Section 2 we will go over the setup and the main ingredients used in the proofs of Theorems 1.1 and 1.5. Section 3 will be devoted to the proof of a more general result that implies Theorems 1.1 and 1.4. We will also prove Theorems 1.5 and 1.8 in Section 3. Finally, in Section 4, we formulate and prove a bilinear Stein-Weiss inequality.

\section{Preliminaries}

We briefly recall some standard notation. A weight, usually denoted by $w, u$, or $v$, is a non-negative locally integrable function. Given a measurable set $E,|E|$ is the Lebesgue measure of $E$ and $w(E)=\int_{E} w d x$ is the weighted measure of $E$. For any measurable function $f$ the average of $f$ over a set $E$ is given by

$$
f_{E} f d x=\frac{1}{|E|} \int_{E} f d x
$$

The euclidian norm of a point $x=\left(x_{1}, \ldots, x_{n}\right) \in \mathbb{R}^{n}$ is given by $|x|=\left(x_{1}^{2}+\right.$ $\left.\cdots+x_{n}^{2}\right)^{1 / 2}$. We will also use the $\ell^{\infty}$ norm $|x|_{\infty}=\max \left(\left|x_{1}\right|, \ldots,\left|x_{n}\right|\right)$. Note that $|x|_{\infty} \leq|x| \leq \sqrt{n}|x|_{\infty}$ for all $x \in \mathbb{R}^{n}$. A cube with center $x_{0}$ and side length $r$, denoted $Q=Q\left(x_{0}, r\right)$, will be all points $x \in \mathbb{R}^{n}$ such that $\left|x-x_{0}\right|_{\infty} \leq \frac{r}{2}$ (occasionally we will use half open cubes as well). For an arbitrary cube $Q, c_{Q}$ will be its center and $\ell(Q)$ its side length, that is, $Q=Q\left(c_{Q}, \ell(Q)\right)$. Given $\lambda>0$ and a cube $Q$ we let $\lambda Q=Q\left(c_{Q}, \lambda \ell(Q)\right)$. The set of dyadic cubes, denoted $\mathscr{D}$, is all cubes of the form $2^{k}\left(m+[0,1)^{n}\right)$ where $k \in \mathbb{Z}$ and $m \in \mathbb{Z}^{n}$. Finally for $k \in \mathbb{Z}$ we let $\mathscr{D}_{k}$ denote the cubes of level $2^{k}$, that is, $\mathscr{D}_{k}=\left\{Q \in \mathscr{D}: \ell(Q)=2^{k}\right\}$. 
2.1. Orlicz spaces and Young functions. We give the necessary details of Orlicz spaces for what follows. For a more detailed account, we encourage the reader to consult [4]. A Young function, $\Phi:[0, \infty) \rightarrow[0, \infty)$, is a continuous, convex, and strictly increasing function, with $\Phi(0)=0$ and $\Phi(t) / t \rightarrow \infty$ as $t \rightarrow \infty$. The main examples that we will be concerned with are of the form $\Phi(t)=t^{r} \log (e+t)^{s}$ for some $r>1$ and $s \in \mathbb{R}$. Given a Young function $\Phi$, there exists another Young function, called the associate function and denoted $\bar{\Phi}$, that satisfies

$$
t \leq \Phi^{-1}(t) \bar{\Phi}^{-1}(t) \leq 2 t, \quad t>0 .
$$

For example, if $p>1$, then the Young function $\Phi_{p}(t)=t^{p}$ has associate Young function $\bar{\Phi}_{p}(t)=\Phi_{p^{\prime}}(t)=t^{p^{\prime}}$ where $1 / p+1 / p^{\prime}=1$. The Orlicz average of $f$ over $Q$ is given by

$$
\|f\|_{\Phi, Q}=\inf \left\{\lambda>0: f_{Q} \Phi\left(\frac{|f(x)|}{\lambda}\right) d x \leq 1\right\} .
$$

In this case we have the generalized Hölder inequality,

$$
f_{Q}|f(x) g(x)| d x \leq 2\|f\|_{\Phi, Q}\|g\|_{\bar{\Phi}, Q} .
$$

For each Young function $\Phi$ the corresponding Orlicz maximal function is defined by

$$
M_{\Phi} f(x)=\sup _{Q \ni x}\|f\|_{\Phi, Q} .
$$

Orlicz maximal functions play an important role in the theory of two weight inequalities. Often a key ingredient is to use $M_{\Phi}: L^{p}\left(\mathbb{R}^{n}\right) \rightarrow L^{p}\left(\mathbb{R}^{n}\right)$ bounds. We state the following characterization due to Pérez [21].

Theorem $\mathbf{C}([21])$. For any $p, 1<p<\infty$,

$$
\left\|M_{\Phi} f\right\|_{L^{p}\left(\mathbb{R}^{n}\right)} \leq C\|f\|_{L^{p}\left(\mathbb{R}^{n}\right)}
$$

if and only if $\Phi$ satisfies the $B_{p}$ integrability condition (denoted $\Phi \in B_{p}$ ): there exists $c>0$ such that

$$
\int_{c}^{\infty} \frac{\Phi(t)}{t^{p}} \frac{d t}{t}<\infty
$$

Typical Young functions that belong to $B_{p}$ usually depend on a parameter. Below are the relevant examples.

Example 2.1. Consider the function $\Phi_{r}(t)=t^{r}$. When $r>1, \Phi_{r}$ is a Young function, and we refer to $\Phi_{r}$ as a power bump. Notice that

$$
\|f\|_{\Phi_{r}, Q}=\left(f_{Q}|f|^{r} d x\right)^{\frac{1}{r}} .
$$

Notice that $\Phi_{r} \in B_{p}$ if and only if $r>p$.

Our second example is more subtle.

Example 2.2. Let $r>1, s \in \mathbb{R}$, and consider the following Young function, called a logarithmic bump function

$$
\Phi(t)=t^{r}[\log (e+t)]^{s} .
$$

We write $\|f\|_{\Phi, Q}=\|f\|_{L^{r}(\log L)^{s}, Q}$ to denote the Orlicz average. The associate Young function is

$$
\bar{\Phi}(t) \approx t^{r^{\prime}}[\log (e+t)]^{-\frac{s}{r-1}} .
$$



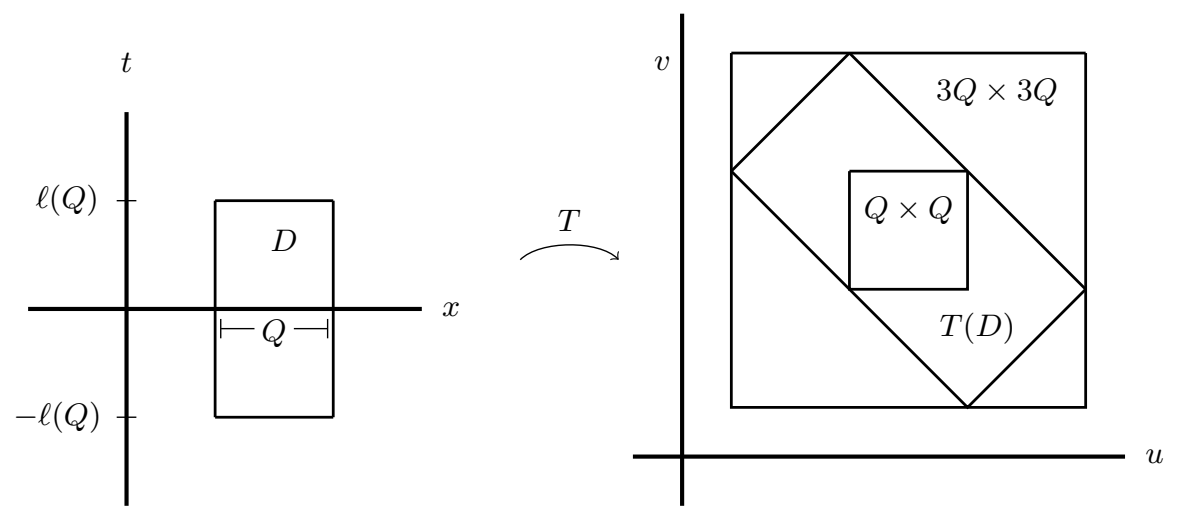

Figure 3 . The change of variable formula, $T(x, t)=(x+t, x-t)$, applied to the rectangle $D=Q \times Q(0,2 \ell(Q))$.

Given $p>1$ in order for $\Phi \in B_{p}$ it suffices to take $r=p$ and $s=-1-\epsilon$ for any $\epsilon>0$. Moreover, one may continue obtaining $B_{p}$ functions by adding more logarithmic terms. For example, the functions

$$
\begin{aligned}
& t^{p} \log (e+t)^{-1-\epsilon}, t^{p} \log (e+t)^{-1} \log \log \left(e^{e}+t\right)^{-1-\epsilon}, \\
& \ldots, t^{p} \log (e+t)^{-1} \cdots \log \cdots \log \left(e^{e^{\cdot}}+t\right)^{-1-\epsilon}, \ldots
\end{aligned}
$$

all belong to $B_{p}$ if $\epsilon>0$. See 4 for a further discussion of logarithmic bumps and the $B_{p}$ condition.

\section{Proof of the Main Results}

We now state our main theorem in the context of the $B_{p}$ condition. By Examples 2.1 and 2.2 following Theorem C. Theorems 1.1 and 1.4 are corollaries of Theorem 3.3 below.

We begin with an auxiliary operator that will play a key role in our analysis. For $r>0$ define,

$$
B_{r}(f, g)(x)=\int_{|t|_{\infty} \leq r} f(x-t) g(x+t) d t .
$$

The operators $B_{2^{k}}$ are used by Kenig and Stein in the analysis of $B I_{\alpha}$. We have the following weighted estimates for $B_{r}$.

Lemma 3.1. Let $0<q \leq 1, w$ be a weight and $Q$ be a cube. Then

$$
\int_{Q} B_{\ell(Q)}(f, g)^{q} w d x \leq C\left(\int_{3 Q} f d x \cdot \int_{3 Q} g d x\right)^{q}\left(\int_{Q} w^{\frac{1}{1-q}} d x\right)^{1-q},
$$

where $\left(\int_{Q} w^{\frac{1}{1-q}} d x\right)^{1-q}=\sup _{Q} w$ if $q=1$.

Proof. By Hölder's inequality with $1 / q$ and $(1 / q)^{\prime}=1 /(1-q)$ we have

$$
\int_{Q} B_{\ell(Q)}(f, g)(x)^{q} w d x \leq\left(\int_{Q} B_{\ell(Q)}(f, g)(x) d x\right)^{q}\left(\int_{Q} w^{\frac{1}{1-q}} d x\right)^{1-q}
$$




$$
=\left(\int_{Q} \int_{|t|_{\infty} \leq \ell(Q)} f(x-t) g(x+t) d t d x\right)^{q}\left(\int_{Q} w^{\frac{1}{1-q}} d x\right)^{1-q} .
$$

We make the change of variables $y=x+t, z=x-t$ in the first integral and notice that if $c_{Q}$ is the center of the cube, then $\left|x-c_{Q}\right|_{\infty} \leq \frac{\ell(Q)}{2}$ and $|t|_{\infty} \leq \ell(Q)$ imply that $(y, z) \in 3 Q \times 3 Q$ (see Figure 3). The lemma follows at once.

Next we consider a discretization of the operator $B I_{\alpha}$ into a dyadic model. Define the dyadic bilinear fractional integral as

$$
B I_{\alpha}^{\mathscr{D}}(f, g)(x)=\sum_{Q \in \mathscr{D}} \frac{|Q|^{\frac{\alpha}{n}}}{|Q|} B_{\ell(Q)}(f, g)(x) \chi_{Q}(x) .
$$

It turns out that $B I_{\alpha}$ is pointwise equivalent to its dyadic model (see [6] for the linear version of Theorem 3.2 below).

Theorem 3.2. If $f, g \geq 0$ are locally integrable, then there exist constants $c$ and $C$, depending on $\alpha$ and $n$, such that

$$
c B I_{\alpha}^{\mathscr{D}}(f, g) \leq B I_{\alpha}(f, g) \leq C B I_{\alpha}^{\mathscr{D}}(f, g) .
$$

Proof. We first discretize the operator $B I_{\alpha}(f, g)$. Notice that $|t| \sim|t|_{\infty}$ and hence

$$
\begin{aligned}
B I_{\alpha}(f, g)(x) & =\sum_{k \in \mathbb{Z}} \int_{2^{k-1}<|t|_{\infty} \leq 2^{k}} \frac{f(x-t) g(x+t)}{|t|^{n-\alpha}} d t \\
& \leq 2^{n-\alpha} \sum_{k \in \mathbb{Z}}\left(2^{-k}\right)^{n-\alpha} \int_{|t|_{\infty} \leq 2^{k}} f(x-t) g(x+t) d t \\
& =c \sum_{k \in \mathbb{Z}} \sum_{Q \in \mathscr{D}_{k}}\left(2^{-k}\right)^{n-\alpha} B_{2^{k}}(f, g)(x) \chi_{Q}(x) \\
& =c \sum_{Q \in \mathscr{D}} \frac{|Q|^{\frac{\alpha}{n}}}{|Q|} B_{\ell(Q)}(f, g)(x) \chi_{Q}(x) .
\end{aligned}
$$

On the other hand, fix $x \in \mathbb{R}^{n}$ and let $\left\{Q_{k}\right\}_{k \in \mathbb{Z}}$ be the unique sequence of dyadic cubes with $x \in Q_{k} \in \mathscr{D}_{k}$. For $N$ consider the truncated version of $B I_{\alpha}^{\mathscr{D}}$ :

$$
\begin{aligned}
& \sum_{\substack{Q \in \mathscr{D} \\
\ell(Q) \leq 2^{N}}} \frac{|Q|^{\frac{\alpha}{n}}}{|Q|} B_{\ell(Q)}(f, g)(x) \chi_{Q}(x) \\
& =\sum_{k=-\infty}^{N} \frac{\left|Q_{k}\right|^{\frac{\alpha}{n}}}{\left|Q_{k}\right|} B_{\ell\left(Q_{k}\right)}(f, g)(x) \\
& =\sum_{k=-\infty}^{N} \frac{\left|Q_{k}\right|^{\frac{\alpha}{n}}}{\left|Q_{k}\right|} \int_{2^{k-1}<|t|_{\infty} \leq 2^{k}} f(x-t) g(x+t) d t \\
& \quad+\sum_{k=-\infty}^{N} \frac{\left|Q_{k}\right|^{\frac{\alpha}{n}}}{\left|Q_{k}\right|} B_{\ell\left(Q_{k-1}\right)}(f, g)(x) \\
& \leq c \sum_{k=-\infty}^{N} \int_{2^{k-1}<|t|_{\infty} \leq 2^{k}} \frac{f(x-t) g(x+t)}{|t|^{n-\alpha}} d t
\end{aligned}
$$




$$
\begin{gathered}
+2^{\alpha-n} \sum_{\substack{Q \in \mathscr{D} \\
\ell(Q) \leq 2^{N}}} \frac{|Q|^{\frac{\alpha}{n}}}{|Q|} B_{\ell(Q)}(f, g)(x) \chi_{Q}(x) \\
\leq c B I_{\alpha}(f, g)(x)+2^{\alpha-n} \sum_{\substack{Q \in \mathscr{D} \\
\ell(Q) \leq 2^{N}}} \frac{|Q|^{\frac{\alpha}{n}}}{|Q|} B_{\ell(Q)}(f, g)(x) \chi_{Q}(x) .
\end{gathered}
$$

Since $\alpha<n$ we may rearrange the terms and take $N \rightarrow \infty$ to obtain

$$
c B I_{\alpha}^{\mathscr{D}}(f, g)(x) \leq B I_{\alpha}(f, g)(x) .
$$

Theorem 3.3. Suppose $1<p_{1}, p_{2}<\infty$ with $1 / p=1 / p_{1}+1 / p_{2}, p \leq q \leq 1$, and $\Phi_{1}, \Phi_{2}$ are Young functions with $\bar{\Phi}_{1} \in B_{p_{1}}$ and $\bar{\Phi}_{2} \in B_{p_{2}}$. If $\left(u, v_{1}, v_{2}\right)$ are weights that satisfy

$$
\sup _{Q}|Q|^{\frac{\alpha}{n}+\frac{1}{q}-\frac{1}{p}}\left(f_{Q} u^{\frac{q}{1-q}}\right)^{\frac{1-q}{q}}\left\|v_{1}^{-1}\right\|_{\Phi_{1}, Q}\left\|v_{2}^{-1}\right\|_{\Phi_{2}, Q}<\infty
$$

where $\left(f_{Q} u^{\frac{q}{1-q}} d x\right)^{\frac{1-q}{q}}=\sup _{Q} u$ for $q=1$, then

$$
B I_{\alpha}: L^{p_{1}}\left(v_{1}^{p_{1}}\right) \times L^{p_{2}}\left(v_{2}^{p_{2}}\right) \rightarrow L^{q}\left(u^{q}\right) .
$$

Proof. By Theorem 3.2 it suffices to work with the dyadic operator $B I_{\alpha}^{\mathscr{D}}$. Since $0<q \leq 1$ it follows that

$$
\int_{\mathbb{R}^{n}}\left(B I_{\alpha}^{\mathscr{D}}(f, g) u\right)^{q} d x \leq c \sum_{Q \in \mathscr{D}} \frac{|Q|^{\frac{\alpha q}{n}}}{|Q|^{q}} \int_{Q} B_{\ell(Q)}(f, g)^{q} u^{q} d x .
$$

By Lemma 3.1 we have

$$
\begin{aligned}
& \int_{\mathbb{R}^{n}}\left(B I_{\alpha}^{\mathscr{D}}(f, g) u\right)^{q} d x \\
& \leq c \sum_{Q \in \mathscr{D}}\left(\frac{|Q|^{\frac{\alpha}{n}}}{|Q|} \int_{3 Q} f d x \cdot \int_{3 Q} g d x\right)^{q}\left(\int_{Q} u^{\frac{q}{1-q}} d x\right)^{1-q} \\
& =c \sum_{Q \in \mathscr{D}}\left(|Q|^{\frac{\alpha}{n}} f_{3 Q} f d x \cdot f_{3 Q} g d x\right)^{q}\left(f_{Q} u^{\frac{q}{1-q}} d x\right)^{1-q}|Q| .
\end{aligned}
$$

We now proceed by following [17, pp. 223-224] (see also [5, 20, 24], for a linear version) to replace the sum over all dyadic cubes to a sum over Calderón-Zygmund cubes. Let

$$
\mathcal{M}_{3 \mathscr{D}}(f, g)(x)=\sup _{x \in Q \in \mathscr{D}} f_{3 Q} f d y \cdot f_{3 Q} g d y,
$$

$a>1$ be a fixed constant to be chosen later, and

$$
\Omega_{k}=\left\{x \in \mathbb{R}^{n}: \mathcal{M}_{3 \mathscr{D}}(f, g)(x)>a^{k}\right\} .
$$

Notice that $\Omega_{k} \supseteq \Omega_{k+1}$, and for each $k \in \mathbb{Z}$ we may write $\Omega_{k}=\bigcup_{j} Q_{j}^{k}$, where $Q_{j}^{k}$ are maximal dyadic cubes that satisfy

$$
f_{3 Q_{j}^{k}} f d y \cdot f_{3 Q_{j}^{k}} g d y>a^{k}
$$


The operator $\mathcal{M}_{3 \mathscr{D}}$ maps $L^{1}\left(\mathbb{R}^{n}\right) \times L^{1}\left(\mathbb{R}^{n}\right)$ into $L^{1 / 2, \infty}\left(\mathbb{R}^{n}\right)$ with a constant, $C_{n}$, that depends only on the dimension. It follows that for a fixed $j, k$ we have

$$
\left|Q_{j}^{k} \cap \Omega_{k+1}\right| \leq \frac{6^{n} C_{n}^{1 / m}}{a^{1 / m}}\left|Q_{j}^{k}\right|
$$

Let $a=6^{m n} 2^{m} C_{n}$ so that $\left|Q_{j}^{k} \cap \Omega_{k+1}\right| \leq \frac{1}{2}\left|Q_{j}^{k}\right|$. If we define $E_{j}^{k}=Q_{j}^{k} \backslash \Omega_{k+1}$, then the family $\left\{E_{j}^{k}\right\}$ is pairwise disjoint and satisfies

$$
\left|E_{j}^{k}\right| \leq\left|Q_{j}^{k}\right| \leq 2\left|E_{j}^{k}\right|
$$

Define

$$
\mathcal{C}_{k}=\left\{Q \in \mathscr{D}: a^{k}<f_{3 Q} f \cdot f_{3 Q} g \leq a^{k+1}\right\} .
$$

We continue by estimating $\int\left(B I_{\alpha}^{\mathscr{D}}(f, g) u\right)^{q}$ picking up with the sum in (12). Notice that every $Q \in \mathscr{D}$ for which the summand in (12) is non-zero belongs to some $\mathcal{C}_{k}$ and every $Q \in \mathcal{C}_{k}$ is contained in a unique $Q_{j}^{k}$. We have

$$
\begin{aligned}
& \sum_{Q \in \mathscr{D}}\left(|Q|^{\frac{\alpha}{n}} f_{3 Q} f d x \cdot f_{3 Q} g d x\right)^{q}\left(f_{Q} u^{\frac{q}{1-q}} d x\right)^{1-q}|Q| \\
& =\sum_{k \in \mathbb{Z}} \sum_{Q \in \mathcal{C}_{k}}\left(|Q|^{\frac{\alpha}{n}} f_{3 Q} f d x \cdot f_{3 Q} g d x\right)^{q}\left(f_{Q} u^{\frac{q}{1-q}} d x\right)^{1-q}|Q| \\
& \leq \sum_{k \in \mathbb{Z}} a^{(k+1) q} \sum_{Q \in \mathcal{C}_{k}}|Q|^{\left(\frac{\alpha}{n}+1\right) q}\left(\int_{Q} u^{\frac{q}{1-q}} d x\right)^{1-q} \\
& \leq \sum_{k \in \mathbb{Z}} a^{(k+1) q} \sum_{j \in \mathbb{Z}} \sum_{Q \subseteq Q_{j}^{k}}|Q|^{\left(\frac{\alpha}{n}+1\right) q}\left(\int_{Q} u^{\frac{q}{1-q}} d x\right)^{1-q} .
\end{aligned}
$$

We now use a packing condition to handle the terms in the innermost sum of (13). Fix a $Q_{j}^{k}$ and consider the sum

$$
\begin{aligned}
& \sum_{Q \subseteq Q_{j}^{k}}|Q|^{\left(\frac{\alpha}{n}+1\right) q}\left(\int_{Q} u^{\frac{q}{1-q}} d x\right)^{1-q} \\
& =\sum_{r=0}^{\infty} \sum_{\substack{Q \subseteq Q_{j}^{k} \\
\ell(Q)=2^{-r} \ell\left(Q_{j}^{k}\right)}}|Q|^{\left(\frac{\alpha}{n}+1\right) q}\left(\int_{Q} u^{\frac{q}{1-q}} d x\right)^{1-q} \\
& =\left|Q_{j}^{k}\right|^{\left(\frac{\alpha}{n}+1\right) q} \sum_{r=0}^{\infty}\left(2^{-r \alpha-r n q}\right) \sum_{\substack{Q \subseteq Q_{j}^{k} \\
\ell(Q)=2^{-r} \ell\left(Q_{j}^{k}\right)}}\left(\int_{Q} u^{\frac{q}{1-q}} d x\right)^{1-q} \\
& \leq\left|Q_{j}^{k}\right|^{\left(\frac{\alpha}{n}+1\right) q} \sum_{r=0}^{\infty}\left(2^{-r \alpha-r n q}\right)\left(\sum_{\substack{Q \subseteq Q_{j}^{k} \\
\ell(Q)=2^{-r} \ell\left(Q_{j}^{k}\right)}} \int_{Q} u^{\frac{q}{1-q}} d x\right)^{1-q}\left(\sum_{\substack{Q \subseteq Q_{j}^{k} \\
\ell(Q)=2^{-r} \ell\left(Q_{j}^{k}\right)}} 1\right)^{q} \\
& =\left|Q_{j}^{k}\right|^{\left(\frac{\alpha}{n}+1\right) q}\left(\int_{Q_{j}^{k}} u^{\frac{q}{1-q}} d x\right)^{1-q} \sum_{r=0}^{\infty} 2^{-r \alpha q}
\end{aligned}
$$




$$
=\frac{2^{\alpha q}}{2^{\alpha q}-1}\left|Q_{j}^{k}\right|^{\frac{\alpha}{n} q}\left(f_{Q_{j}^{k}} u^{\frac{q}{1-q}} d x\right)^{1-q}\left|Q_{j}^{k}\right| .
$$

Using this inequality in (13) we have

$$
\begin{aligned}
& \int_{\mathbb{R}^{n}}\left(B I_{\alpha}^{\mathscr{D}}(f, g) u\right)^{q} d x \\
& \leq c \sum_{j, k}\left(\left|Q_{j}^{k}\right|^{\frac{\alpha}{n}} f_{3 Q_{j}^{k}} f d x \cdot f_{3 Q_{j}^{k}} g d x\right)^{q}\left(f_{Q_{j}^{k}} u^{\frac{q}{1-q}} d x\right)^{1-q}\left|Q_{j}^{k}\right| .
\end{aligned}
$$

We replace the cubes $Q_{j}^{k}$ by $3 Q_{j}^{k}$ as need be, use the generalized Hölder inequality on both the terms involving $f$ and $g$, and use condition (11) to continue. We have the sum in (14) bounded by

$$
\begin{aligned}
& \leq c \sum_{j, k}\left(\left|3 Q_{j}^{k}\right|^{\frac{\alpha}{n}}\left\|v_{1}^{-1}\right\|_{\Phi_{1}, 3 Q_{j}^{k}}\left\|v_{2}^{-1}\right\|_{\Phi_{2}, 3 Q_{j}^{k}}\left\|f v_{1}\right\|_{\bar{\Phi}_{1}, 3 Q_{j}^{k}}\left\|g v_{2}\right\|_{\bar{\Phi}_{2}, 3 Q_{j}^{k}}\right)^{q} \\
& \quad \times\left(f_{3 Q_{j}^{k}} u^{\frac{q}{1-q}} d x\right)^{1-q}\left|Q_{j}^{k}\right| \\
& \leq c \sum_{j, k}\left(\left\|f v_{1}\right\|_{\bar{\Phi}_{1}, 3 Q_{j}^{k}}\left\|g v_{2}\right\|_{\bar{\Phi}_{2}, 3 Q_{j}^{k}}\right)^{q}\left|Q_{j}^{k}\right|^{\frac{q}{p}}
\end{aligned}
$$

We make our final estimates using the steps below in the following order:

1. the fact that $p \leq q$,

2. a discrete Hölder inequality with $p_{1} / p$ and $p_{2} / p$,

3. $\left|Q_{j}^{k}\right| \leq 2\left|E_{j}^{k}\right|$,

4. the family $\left\{E_{j}^{k}\right\}$ is pairwise disjoint,

5. the maximal functions $M_{\bar{\Phi}_{1}}$ and $M_{\bar{\Phi}_{2}}$ are bounded on $L^{p_{1}}\left(\mathbb{R}^{n}\right)$ and $L^{p_{2}}\left(\mathbb{R}^{n}\right)$ respectively.

Finally we have,

$$
\begin{aligned}
& \int_{\mathbb{R}^{n}}\left(B I_{\alpha}^{\mathscr{D}}(f, g) u\right)^{q} d x \\
& \leq c \sum_{j, k}\left(\left\|f v_{1}\right\|_{\bar{\Phi}_{1}, 3 Q_{j}^{k}}\left\|g v_{2}\right\|_{\bar{\Phi}_{2}, 3 Q_{j}^{k}}\right)^{q}\left|Q_{j}^{k}\right|^{\frac{q}{p}} . \\
& \leq c\left(\sum_{j, k}\left(\left\|f v_{1}\right\|_{\bar{\Phi}_{1}, 3 Q_{j}^{k}}\left\|g v_{2}\right\|_{\bar{\Phi}_{2}, 3 Q_{j}^{k}}\right)^{p}\left|E_{j}^{k}\right|\right)^{\frac{q}{p}} \\
& \leq c\left(\sum_{j, k}\left\|f v_{1}\right\|_{\bar{\Phi}_{1}, 3 Q_{j}^{k}}^{p_{1}}\left|E_{j}^{k}\right|\right)^{\frac{q}{p_{1}}}\left(\sum_{j, k}\left\|g v_{2}\right\|_{\bar{\Phi}_{2}, 3 Q_{j}^{k}}^{p_{2}}\left|E_{j}^{k}\right|\right)^{\frac{q}{p_{2}}} \\
& \leq c\left(\sum_{j, k} \int_{E_{j}^{k}} M_{\bar{\Phi}_{1}}\left(f v_{1}\right)^{p_{1}} d x\right)^{\frac{q}{p_{1}}}\left(\sum_{j, k} \int_{E_{j}^{k}} M_{\bar{\Phi}_{2}}\left(g v_{2}\right)^{p_{2}} d x\right)^{\frac{q}{p_{2}}}
\end{aligned}
$$




$$
\begin{aligned}
& \leq c\left(\int_{\mathbb{R}^{n}} M_{\bar{\Phi}_{1}}\left(f v_{1}\right)^{p_{1}} d x\right)^{\frac{q}{p_{1}}}\left(\int_{\mathbb{R}^{n}} M_{\bar{\Phi}_{2}}\left(g v_{2}\right)^{p_{2}} d x\right)^{\frac{q}{p_{2}}} \\
& \leq c\left(\int_{\mathbb{R}^{n}}\left(f v_{1}\right)^{p_{1}} d x\right)^{\frac{q}{p_{1}}}\left(\int_{\mathbb{R}^{n}}\left(g v_{2}\right)^{p_{2}} d x\right)^{\frac{q}{p_{2}}} .
\end{aligned}
$$

Remark 3.4. One can trace the bounds on the constants to see that

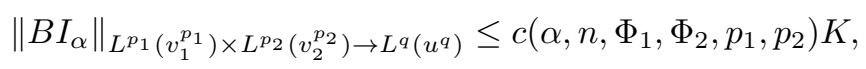

where $K$ is the constant from (11). The dependence on $K$ also follows from a variation of the scaling argument given in [5]. Indeed, notice that the constant $K=K\left(u, v_{1}, v_{2}\right)$ scales as follows: for $\lambda, \gamma>0$,

$$
K\left(\lambda u, \gamma v_{1}, v_{2}\right)=\lambda \gamma^{-1} K\left(u, v_{1}, v_{2}\right) .
$$

Meanwhile, if we consider the inequality

$$
\left\|B I_{\alpha}\right\|_{L^{p_{1}}\left(\left(\gamma v_{1}\right)^{p_{1}}\right) \times L^{p_{2}}\left(v_{2}^{p_{2}}\right) \rightarrow L^{q}\left((\lambda u)^{q}\right)} \leq \Theta\left(K\left(\lambda u, \gamma v_{1}, v_{2}\right)\right)
$$

we see that

$$
\left\|B I_{\alpha}\right\|_{L^{p_{1}}\left(\left(\gamma v_{1}\right)^{p_{1}}\right) \times L^{p_{2}}\left(v_{2}^{p_{2}}\right) \rightarrow L^{q}\left((\lambda u)^{q}\right)}=\frac{\lambda}{\gamma}\left\|B I_{\alpha}\right\|_{L^{p_{1}}\left(v_{1}^{p_{1}}\right) \times L^{p_{2}}\left(v_{2}^{p_{2}}\right) \rightarrow L^{q}\left(u^{q}\right)} .
$$

By taking $\lambda=1$ and $\gamma=K$ we see that (15) holds.

Theorem 1.5 is now essentially a corollary of Theorem 1.1

Proof of Theorem 1.5. Suppose that $1<p_{1}, p_{2}<\infty$ are such that $1<1 / q=$ $1 / p_{1}+1 / p_{2}-\alpha / n, \tilde{q}=q /(1-q)$ (note: $\left.1<\tilde{q}<\infty\right)$ and $\left(w_{1}, w_{2}\right) \in A_{\left(p_{1}, p_{2}\right), \tilde{q}}$, i.e., condition (9) holds. If $u=w_{1} w_{2}, v_{1}=w_{1}$, and $v_{2}=w_{2}$, then condition (8) becomes

$$
\sup _{Q}\left(f_{Q}\left(w_{1} w_{2}\right)^{\frac{q}{1-q}} d x\right)^{\frac{1-q}{q}}\left(f_{Q} w_{1}^{-r p_{1}^{\prime}} d x\right)^{\frac{1}{r p_{1}^{\prime}}}\left(f_{Q} w_{2}^{-r p_{2}^{\prime}} d x\right)^{\frac{1}{r p_{2}^{\prime}}}<\infty
$$

for some $r>1$. Since $\left(w_{1}, w_{2}\right) \in A_{\left(p_{1}, p_{2}\right), \tilde{q}}$ by Theorem 3.4 in [17] we have $w_{1}^{-p_{1}^{\prime}} \in$ $A_{2 p_{1}^{\prime}}$ and $w_{2}^{-p_{2}^{\prime}} \in A_{2 p_{2}^{\prime}}$. In particular the weights $w_{1}^{-p_{1}^{\prime}}, w_{2}^{-p_{2}^{\prime}}$ each satisfy a reverse Hölder inequality: there exists an $r>1$ such that

$$
\left(f_{Q} w_{1}^{-r p_{1}^{\prime}} d x\right)^{\frac{1}{r}} \leq C f_{Q} w_{1}^{-p_{1}^{\prime}} d x \text { and }\left(f_{Q} w_{2}^{-r p_{2}^{\prime}} d x\right)^{\frac{1}{r}} \leq C f_{Q} w_{1}^{-p_{2}^{\prime}} d x .
$$

Using this fact we have that condition (8) is satisfied, so by Theorem 1.1 ,

$$
\begin{aligned}
& \left(\int_{\mathbb{R}^{n}}\left(B I_{\alpha}(f, g) w_{1} w_{2}\right)^{q} d x\right)^{\frac{1}{q}} \\
& \quad \leq C\left(\int_{\mathbb{R}^{n}}\left(f w_{1}\right)^{p_{1}} d x\right)^{\frac{1}{p_{1}}}\left(\int_{\mathbb{R}^{n}}\left(g w_{2}\right)^{p_{2}} d x\right)^{\frac{1}{p_{2}}} .
\end{aligned}
$$


We now reap the benefits of our techniques to obtain some interesting estimates involving the bilinear operators $\mathcal{M}_{\alpha}, B I_{\alpha}$, and $B M_{\alpha}$. We start with a precursor for Theorem 1.8 relating $B I_{\alpha}, \mathcal{M}_{\alpha}$, and the Hardy-Littlewood maximal function applied to the weight.

Theorem 3.5. Suppose $w$ is a locally integrable function and $0<q<1$. Then

$$
\int_{\mathbb{R}^{n}} B I_{\alpha}(f, g)^{q} w d x \leq C \int_{\mathbb{R}^{n}} \mathcal{M}_{\alpha}(f, g)^{q} M\left(w^{\frac{1}{1-q}}\right)^{1-q} d x .
$$

Proof. Let $\left\{Q_{j}^{k}\right\}$ and $\left\{E_{j}^{k}\right\}$ be the families of sets from the proof of Theorem 3.3 . We begin with inequality (14) with $u=w^{1 / q}$ :

$$
\begin{aligned}
& \int_{\mathbb{R}^{n}} B I_{\alpha}(f, g)^{q} w d x \\
& \leq c \sum_{j, k}\left(\left|Q_{j}^{k}\right|^{\frac{\alpha}{n}} f_{3 Q_{j}^{k}} f d x \cdot f_{3 Q_{j}^{k}} g d x\right)^{q}\left(f_{Q_{j}^{k}} w^{\frac{1}{1-q}} d x\right)^{1-q}\left|Q_{j}^{k}\right| \\
& \leq c \sum_{j, k}\left(\left|Q_{j}^{k}\right|^{\frac{\alpha}{n}} f_{3 Q_{j}^{k}} f d x \cdot f_{3 Q_{j}^{k}} g d x\right)^{q}\left(f_{Q_{j}^{k}} w^{\frac{1}{1-q}} d x\right)^{1-q}\left|E_{j}^{k}\right| \\
& \leq c \sum_{j, k} \int_{E_{j}^{k}} \mathcal{M}_{\alpha}(f, g) M\left(w^{\frac{1}{1-q}}\right)^{1-q} d x \\
& \leq c \int_{\mathbb{R}^{n}} \mathcal{M}_{\alpha}(f, g)^{q} M\left(w^{\frac{1}{1-q}}\right)^{1-q} d x .
\end{aligned}
$$

Using similar methods we prove Theorem 1.8

Proof of Theorem 1.8. Using the exact same decomposition as above we have

$$
\begin{aligned}
& \int_{\mathbb{R}^{n}} B I_{\alpha}(f, g)^{q} w d x \\
& \leq c \sum_{j, k}\left(\left|Q_{j}^{k}\right|^{\frac{\alpha}{n}} f_{3 Q_{j}^{k}} f d x \cdot f_{3 Q_{j}^{k}} g d x\right)^{q}\left(f_{Q_{j}^{k}} w^{\frac{1}{1-q}} d x\right)^{1-q}\left|Q_{j}^{k}\right| \\
& \leq c \sum_{j, k}\left(\left|Q_{j}^{k}\right|^{\frac{\alpha}{n}} f_{3 Q_{j}^{k}} f d x \cdot f_{3 Q_{j}^{k}} g d x\right)^{q} w\left(Q_{j}^{k}\right),
\end{aligned}
$$

where we used the fact that $w \in R H_{(1 / q)^{\prime}}$ in the last inequality. Since $w \in R H_{(1 / q)^{\prime}}$ it belongs to $A_{\infty}$, and so there is a constant such that $w\left(Q_{j}^{k}\right) \leq c w\left(E_{j}^{k}\right)$. Continuing with the estimates we have

$$
\begin{aligned}
& \leq c \sum_{j, k}\left(\left|Q_{j}^{k}\right|^{\frac{\alpha}{n}} f_{3 Q_{j}^{k}} f d x \cdot f_{3 Q_{j}^{k}} g d x\right)^{q} w\left(E_{j}^{k}\right) \\
& \leq c \sum_{j, k} \int_{E_{j}^{k}} \mathcal{M}_{\alpha}(f, g)^{q} w d x \\
& \leq c \int_{\mathbb{R}^{n}} \mathcal{M}_{\alpha}(f, g)^{q} w d x .
\end{aligned}
$$




\section{EXAMPLES AND NECESSARY CONDITIONS}

4.1. A bilinear Stein-Weiss inequality. Given $0<\alpha<n$ let $T_{\alpha}$ be defined by

$$
T_{\alpha} f(x)=I_{n-\alpha} f(x)=\int_{\mathbb{R}^{n}} \frac{f(y)}{|x-y|^{\alpha}} d y .
$$

Stein and Weiss [25] proved the following weighted inequality for $T_{\alpha}$ :

$$
\left(\int_{\mathbb{R}^{n}}\left(\frac{T_{\alpha} f(x)}{|x|^{\beta}}\right)^{q} d x\right)^{1 / q} \leq C\left(\int_{\mathbb{R}^{n}}\left(f(x)|x|^{\gamma}\right)^{p} d x\right)^{1 / p},
$$

where

$$
\begin{gathered}
\beta<\frac{n}{q}, \quad \gamma<\frac{n}{p^{\prime}}, \\
\alpha+\beta+\gamma=n+\frac{n}{q}-\frac{n}{p}, \\
\beta+\gamma \geq 0 .
\end{gathered}
$$

Conditions (16), (17), and (18) are actually sharp. Condition (16) ensures that $|x|^{-\beta q}$ and $|x|^{-\gamma p^{\prime}}$ are locally integrable. Condition (17) follows from a scaling argument and condition (18) is a necessary condition for the weights to satisfy a general two weight inequality (see 24]).

Below, we prove a bilinear Stein-Weiss inequality. For $0<\alpha<n$ let $B T_{\alpha}$ be the bilinear operator defined by

$$
B T_{\alpha}(f, g)(x)=B I_{n-\alpha}(f, g)(x)=\int_{\mathbb{R}^{n}} \frac{f(x-t) g(x+t)}{|t|^{\alpha}} d t .
$$

Theorem 4.1. Suppose $1<p_{1}, p_{2}<\infty$ and $q$ satisfy $p \leq q \leq 1$. If $\alpha, \beta, \gamma_{1}, \gamma_{2}$ satisfy the conditions

$$
\left\{\begin{array}{c}
\beta<n\left(\frac{1}{q}-1\right), \quad \gamma_{1}<\frac{n}{p_{1}^{\prime}}, \quad \gamma_{2}<\frac{n}{p_{2}^{\prime}} \\
\alpha+\beta+\gamma_{1}+\gamma_{2}=n+\frac{n}{q}-\frac{n}{p} \\
\beta+\gamma_{1}+\gamma_{2} \geq 0
\end{array}\right.
$$

then the following inequality holds for all $f, g \geq 0$ :

$$
\begin{aligned}
& \left(\int_{\mathbb{R}^{n}}\left(\frac{B T_{\alpha}(f, g)(x)}{|x|^{\beta}}\right)^{q} d x\right)^{1 / q} \\
& \quad \leq C\left(\int_{\mathbb{R}^{n}}\left(f(x)|x|^{\gamma_{1}}\right)^{p_{1}} d x\right)^{1 / p_{1}}\left(\int_{\mathbb{R}^{n}}\left(g(x)|x|^{\gamma_{2}}\right)^{p_{2}} d x\right)^{1 / p_{2}} .
\end{aligned}
$$

Proof. By Theorem 1.1 we need to prove that their exist $r>1$ and a constant $C$ such that

$$
|Q|^{1-\frac{\alpha}{n}+\frac{1}{q}-\frac{1}{p}}\left(f_{Q}|x|^{-\frac{\beta q}{1-q}} d x\right)^{\frac{1-q}{q}} \prod_{i=1}^{2}\left(f_{Q}|x|^{-\gamma_{i} r p_{i}^{\prime}} d x\right)^{\frac{1}{r p_{i}^{\prime}}} \leq C
$$

for all cubes $Q$. From here we follow the standard estimates for power weights. Let $r>1$ be such that $r \gamma_{1}<n / p_{1}^{\prime}$ and $r \gamma_{2}<n / p_{2}^{\prime}$. Given a cube $Q$ let $Q_{0}$ be its translate to the origin, i.e., $Q_{0}=Q(0, \ell(Q))$. Then either $2 Q_{0} \cap Q=\varnothing$ or 
$2 Q_{0} \cap Q \neq \varnothing$. In the case $2 Q_{0} \cap Q=\varnothing$ we have $\left|c_{Q}\right|_{\infty} \geq \ell(Q)$ and $|x| \sim|x|_{\infty} \sim$ $\left|c_{Q}\right|_{\infty} \neq 0$ for all $x \in Q$. Using this fact we have

$$
\begin{gathered}
|Q|^{1-\frac{\alpha}{n}+\frac{1}{q}-\frac{1}{p}}\left(f_{Q}|x|^{-\frac{\beta q}{1-q}} d x\right)^{\frac{1-q}{q}} \prod_{i=1}^{2}\left(f_{Q}|x|^{-\gamma_{i} r p_{i}^{\prime}} d x\right)^{\frac{1}{r p_{i}^{\prime}}} \\
=\ell(Q)^{\beta+\gamma_{1}+\gamma_{2}}\left(f_{Q}|x|^{-\frac{\beta q}{1-q}} d x\right)^{\frac{1-q}{q}} \prod_{i=1}^{2}\left(f_{Q}|x|^{-\gamma_{i} r p_{i}^{\prime}} d x\right)^{\frac{1}{r p_{i}^{\prime}}} \\
\leq c \ell(Q)^{\beta+\gamma_{1}+\gamma_{2}}\left|c_{Q}\right|_{\infty}^{-\beta-\gamma_{1}-\gamma_{2}} \leq C,
\end{gathered}
$$

where in the first line we have used the second equality in (19) and in the last estimate we have used the third inequality in (19). When $2 Q_{0} \cap Q \neq \varnothing$ we have that $Q \subseteq B=B(0,5 \ell(Q))$, the euclidean ball of radius $5 \ell(Q)$ about the origin. Thus,

$$
\begin{aligned}
& |Q|^{1-\frac{\alpha}{n}+\frac{1}{q}-\frac{1}{p}}\left(f_{Q}|x|^{-\frac{\beta q}{1-q}} d x\right)^{\frac{1-q}{q}} \prod_{i=1}^{2}\left(f_{Q}|x|^{-\gamma_{i} r p_{i}^{\prime}} d x\right)^{\frac{1}{r p_{i}^{\prime}}} \\
& \leq c \ell(Q)^{\beta+\gamma_{1}+\gamma_{2}}\left(f_{B}|x|^{-\frac{\beta q}{1-q}} d x\right)^{\frac{1-q}{q}} \prod_{i=1}^{2}\left(f_{B}|x|^{-\gamma_{i} r p_{i}^{\prime}} d x\right)^{\frac{1}{r p_{i}^{\prime}}} \\
& \leq C .
\end{aligned}
$$

4.2. Necessary conditions. Apparently our techniques do not address the case $q>1$. That is, other than the trivial conditions mentioned in the introduction, we do not know of sufficient conditions on weights $\left(u, v_{1}, v_{1}\right)$ that imply

$$
B I_{\alpha}: L^{p_{1}}\left(v_{1}^{p_{1}}\right) \times L^{p_{2}}\left(v_{2}^{p_{2}}\right) \rightarrow L^{q}\left(u^{q}\right)
$$

when $q>1$. Here we present a necessary condition for the two weight inequality for $B M_{\alpha}$, which in turn is necessary for $B I_{\alpha}$ when $0<\alpha<n$.

Theorem 4.2. Suppose $0 \leq \alpha<n, 1<p_{1}, p_{2}<\infty, q \geq 1$ and

$$
B M_{\alpha}: L^{p_{1}}\left(v_{1}^{p_{1}}\right) \times L^{p_{2}}\left(v_{2}^{p_{2}}\right) \rightarrow L^{q}\left(u^{q}\right) .
$$

Then there exists a constant $C$ such that

$$
|Q|^{\frac{\alpha}{n}+\frac{1}{q}-\frac{1}{p}}\left(\inf _{Q} u\right)\left(f_{Q} v_{1}^{-p_{1}^{\prime}} d x\right)^{\frac{1}{p_{1}^{\prime}}}\left(f_{Q} v_{2}^{-p_{2}^{\prime}} d x\right)^{\frac{1}{p_{2}^{\prime}}} \leq C
$$

for all cubes $Q$.

When $q=1$, compare (20) with our sufficient condition:

$$
|Q|^{\frac{\alpha}{n}+\frac{1}{q}-\frac{1}{p}}\left(\sup _{Q} u\right)\left(f_{Q} v_{1}^{-r p_{1}^{\prime}} d x\right)^{\frac{1}{r p_{1}^{\prime}}}\left(f_{Q} v_{2}^{-r p_{2}^{\prime}} d x\right)^{\frac{1}{r p_{2}^{\prime}}} \leq C .
$$

Proof. Fix a cube $Q=Q\left(c_{Q}, \ell(Q)\right)$ and let $f=\chi_{Q} v_{1}^{-p_{1}^{\prime}}, g=\chi_{Q} v_{2}^{-p_{2}^{\prime}}$. Plugging these functions into $B M_{\alpha}$ we have

$$
\left(\int_{Q}\left(B M_{\alpha}(f, g) u\right)^{q} d x\right)^{\frac{1}{q}} \leq\left(\int_{\mathbb{R}^{n}}\left(B M_{\alpha}(f, g) u\right)^{q} d x\right)^{\frac{1}{q}}
$$




$$
\begin{aligned}
& \leq C\left(\int_{\mathbb{R}^{n}}\left(f v_{1}\right)^{p_{1}} d x\right)^{\frac{1}{p_{1}}}\left(\int_{\mathbb{R}^{n}}\left(g v_{2}\right)^{p_{2}} d x\right)^{\frac{1}{p_{2}}} \\
& =C\left(\int_{Q} v_{1}^{-p_{1}^{\prime}} d x\right)^{\frac{1}{p_{1}}}\left(\int_{Q} v_{2}^{-p_{2}^{\prime}} d x\right)^{\frac{1}{p_{2}}} .
\end{aligned}
$$

We now work with the leftmost quantity:

$$
\begin{aligned}
& \left(\int_{Q}\left(B M_{\alpha}(f, g) u\right)^{q} d x\right)^{\frac{1}{q}} \\
& \geq c\left(\inf _{Q} u\right)\left(\int_{Q}\left(\frac{|Q|^{\frac{\alpha}{n}}}{|Q|} \int_{|t|_{\infty} \leq \ell(Q)} f(x-t) g(x+t) d t\right)^{q} d x\right)^{\frac{1}{q}} \\
& \geq c\left(\inf _{Q} u\right)|Q|^{\frac{\alpha}{n}+\frac{1}{q}} \frac{1}{|Q|^{2}} \int_{Q} \int_{|t|_{\infty} \leq \ell(Q)} f(x-t) g(x+t) d t d x .
\end{aligned}
$$

We now make the same change of variables as in Lemma 3.1 $y=x+t, z=x-t$. If $(y, z) \in Q \times Q$, then $x=\frac{y+z}{2} \in Q$ and $t=\frac{z-y}{2}$ satisfies $|t|_{\infty} \leq \ell(Q)$. Indeed

$$
\left|x-c_{Q}\right|_{\infty}=\left|\frac{y+z}{2}-c_{Q}\right|_{\infty} \leq \frac{\left|y-c_{Q}\right|_{\infty}}{2}+\frac{\left|z-c_{Q}\right|_{\infty}}{2} \leq \frac{\ell(Q)}{2}
$$

and

$$
|t|_{\infty}=\frac{|y-z|_{\infty}}{2} \leq \frac{\left|y-c_{Q}\right|_{\infty}}{2}+\frac{\left|c_{Q}-z\right|_{\infty}}{2} \leq \frac{\ell(Q)}{2} \leq \ell(Q) .
$$

For a geometric interpretation (see Figure 3), notice that $Q \times Q \subseteq T(Q \times Q(0,2 \ell(Q)))$, where $T(x, t)=(x-t, x+t)=(y, z)$. This implies that

$$
\begin{array}{r}
\int_{Q} \int_{|t|_{\infty} \leq \ell(Q)} f(x-t) g(x+t) d t d x \geq c \int_{Q} \int_{Q} f(y) g(z) d y d z \\
=c\left(\int_{Q} v_{1}^{-p_{1}^{\prime}} d x\right)\left(\int_{Q} v_{2}^{-p_{2}^{\prime}} d x\right) .
\end{array}
$$

Combining everything we have

$$
\begin{aligned}
&|Q|^{\frac{\alpha}{n}+\frac{1}{q}}\left(\inf _{Q} u\right)\left(f_{Q} v_{1}^{-p_{1}^{\prime}} d x\right)\left(f_{Q} v_{2}^{-p_{2}^{\prime}} d x\right) \\
& \leq C\left(\int_{Q} v_{1}^{-p_{1}^{\prime}} d x\right)^{\frac{1}{p_{1}}}\left(\int_{Q} v_{2}^{-p_{2}^{\prime}} d x\right)^{\frac{1}{p_{2}}},
\end{aligned}
$$

which yields (20).

\section{REFERENCES}

[1] A. Bernardis, O. Gorosito, and G. Pradolini, Weighted inequalities for multilinear potential operators and its commutators, preprint http://arxiv.org/abs/1007.0445.

[2] F. Bernicot, D. Maldonado, K. Moen, and V. Naibo, Bilinear Sobolev-Poincaré inequalities and Leibniz-type rules, J. Geom. Anal. (2012), DOI: 10.1007/s12220-012-9367-4.

[3] Xi Chen and Qingying Xue, Weighted estimates for a class of multilinear fractional type operators, J. Math. Anal. Appl. 362 (2010), no. 2, 355-373, DOI 10.1016/j.jmaa.2009.08.022. MR2557692(2010i:42036)

[4] David V. Cruz-Uribe, José Maria Martell, and Carlos Pérez, Weights, extrapolation and the theory of Rubio de Francia, Operator Theory: Advances and Applications, vol. 215, Birkhäuser/Springer Basel AG, Basel, 2011. MR2797562 (2012f:42001) 
[5] David Cruz-Uribe and Kabe Moen, Sharp norm inequalities for commutators of classical operators, Publ. Mat. 56 (2012), no. 1, 147-190, DOI 10.5565/PUBLMAT_56112_06. MR2918187

[6] D. Cruz-Uribe and K. Moen, One and two weight norm inequalities for Riesz potentials Illinois J. Math. to appear.

[7] Yong Ding and Chin-Cheng Lin, Rough bilinear fractional integrals, Math. Nachr. 246/247 (2002), 47-52, DOI 10.1002/1522-2616(200212)246:1〈47::AID-MANA47〉3.0.CO;27. MR1944548 (2003j:42019)

[8] Loukas Grafakos, On multilinear fractional integrals, Studia Math. 102 (1992), no. 1, 49-56. MR $1164632(93 \mathrm{~d}: 42021)$

[9] Loukas Grafakos and Nigel Kalton, Some remarks on multilinear maps and interpolation, Math. Ann. 319 (2001), no. 1, 151-180, DOI 10.1007/PL00004426. MR.1812822 (2002a:46032)

[10] Takeshi Iida, Yasuo Komori-Furuya, and Enji Sato, A note on multilinear fractional integrals, Anal. Theory Appl. 26 (2010), no. 4, 301-307, DOI 10.1007/s10496-010-0301-y. MR2770468 (2011m:42024)

[11] Carlos E. Kenig and Elias M. Stein, Multilinear estimates and fractional integration, Math. Res. Lett. 6 (1999), no. 1, 1-15. MR.1682725 (2000k:42023a)

[12] Michael T. Lacey, The bilinear maximal functions map into $L^{p}$ for $2 / 3<p \leq 1$, Ann. of Math. (2) 151 (2000), no. 1, 35-57, DOI 10.2307/121111. MR.1745019(2001b:42015)

[13] Michael Lacey and Christoph Thiele, $L^{p}$ estimates on the bilinear Hilbert transform for $2<p<\infty$, Ann. of Math. (2) 146 (1997), no. 3, 693-724, DOI 10.2307/2952458. MR1491450 (99b:42014)

[14] Michael Lacey and Christoph Thiele, On Calderón's conjecture, Ann. of Math. (2) 149 (1999), no. 2, 475-496, DOI 10.2307/120971. MR1689336 (2000d:42003)

[15] Andrei K. Lerner, Sheldy Ombrosi, Carlos Pérez, Rodolfo H. Torres, and Rodrigo TrujilloGonzález, New maximal functions and multiple weights for the multilinear CalderónZygmund theory, Adv. Math. 220 (2009), no. 4, 1222-1264, DOI 10.1016/j.aim.2008.10.014. MR2483720(2010f:42024)

[16] Diego Maldonado, Kabe Moen, and Virginia Naibo, Weighted multilinear Poincaré inequalities for vector fields of Hörmander type, Indiana Univ. Math. J. 60 (2011), no. 2, 473-506, DOI 10.1512/iumj.2011.60.4156. MR2963782

[17] Kabe Moen, Weighted inequalities for multilinear fractional integral operators, Collect. Math. 60 (2009), no. 2, 213-238, DOI 10.1007/BF03191210. MR2514845(2010d:42028)

[18] K. Moen and V. Naibo, Higher-order multilinear Poincaré and Sobolev inequalities in Carnot groups, submitted (2010); http://arxiv.org/abs/1008.0414.

[19] Benjamin Muckenhoupt and Richard Wheeden, Weighted norm inequalities for fractional integrals, Trans. Amer. Math. Soc. 192 (1974), 261-274. MR0340523 (49 \#5275)

[20] Carlos Pérez, Two weighted inequalities for potential and fractional type maximal operators, Indiana Univ. Math. J. 43 (1994), no. 2, 663-683, DOI 10.1512/iumj.1994.43.43028. MR.1291534 (95m:42028)

[21] C. Pérez, On sufficient conditions for the boundedness of the Hardy-Littlewood maximal operator between weighted $L^{p}$-spaces with different weights, Proc. London Math. Soc. (3) 71 (1995), no. 1, 135-157, DOI 10.1112/plms/s3-71.1.135. MR.1327936 (96k:42023)

[22] Gladis Pradolini, Weighted inequalities and pointwise estimates for the multilinear fractional integral and maximal operators, J. Math. Anal. Appl. 367 (2010), no. 2, 640-656, DOI 10.1016/j.jmaa.2010.02.008. MR2607287 (2011c:42051)

[23] Eric T. Sawyer, A characterization of two weight norm inequalities for fractional and Poisson integrals, Trans. Amer. Math. Soc. 308 (1988), no. 2, 533-545, DOI 10.2307/2001090. MR930072 (89d:26009)

[24] E. Sawyer and R. L. Wheeden, Weighted inequalities for fractional integrals on Euclidean and homogeneous spaces, Amer. J. Math. 114 (1992), no. 4, 813-874, DOI 10.2307/2374799. MR 1175693 (94i:42024)

[25] E. M. Stein and Guido Weiss, Fractional integrals on $n$-dimensional Euclidean space, J. Math. Mech. 7 (1958), 503-514. MR0098285 (20 \#4746) 0350

Department of Mathematics, University of Alabama, Tuscaloosa, Alabama 35487 - 